\title{
Gobierno electrónico
}

Análisis de los conceptos de tecnología, comodidad y democracia

Joaquín Yrivarren Espinoza

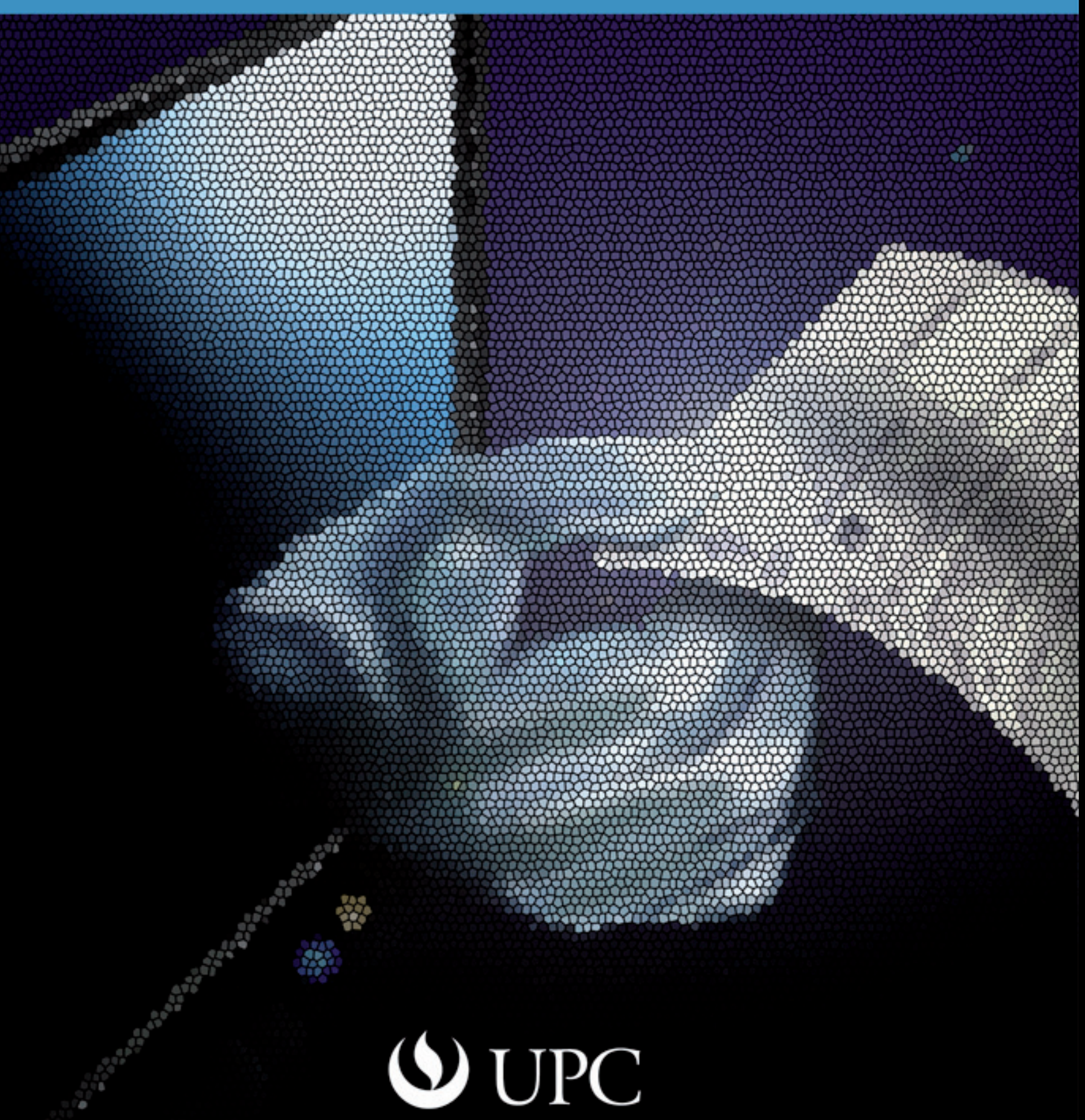




\section{Gobierno electrónico}

Análisis de los conceptos de tecnología, comodidad y democracia

Joaquín Yrivarren Espinoza

Lima, julio de 2011

$\boldsymbol{\omega}$ UPC 
(C) Universidad Peruana de Ciencias Aplicadas (UPC)

Primera publicación: julio de 2011

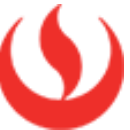

Impreso en el Perú - Printed in Peru

Cubierta:

Germán Ruiz Ch.

Corrección de estilo: Jorge Coaguila

Diseño de cubierta: Germán Ruiz Ch.

Diagramación: Diana Patrón

Editor del proyecto editorial

Universidad Peruana de Ciencias Aplicadas S. A. C.

Av. Alonso de Molina 1611, Lima 33, Perú.

Teléf. 313-3333

www.upc.edu.pe

Primera edición: julio de 2011

Digitalizado y Distribuido por YoPublico S.A.C.

\section{2 yopublico}

www.yopublico.net

Telf: 51-1-221 9998

Dirección: Av. 2 de Mayo 534 Of. 304,

Miraflores Lima-Perú

\section{Universidad Peruana de Ciencias Aplicadas (UPC) Centro de Información}

Yrivarren Espinoza, Joaquín Rodrigo. Gobierno electrónico. Análisis de los

conceptos de tecnología, comodidad y democracia

Lima: Universidad Peruana de Ciencias Aplicadas (UPC), 2015

ISBN de la versión impresa: 978-612-4041-55-6

ISBN de la versión PDF: 978-612-4041-64-8

ISBN de la versión e-Pub: 978-612-4191-85-5

GOBIERNO ELECTRÓNICO, VOTO ELECTRÓNICO, COMUNICACIÓN EN LA

ADMINISTRACIÓN PÚBLICA, GOBIERNO LOCAL, MIRAFLORES

351.02854678 YRIV

Todos los derechos reservados. Esta publicación no puede ser reproducida, ni en todo ni en parte, ni registrada en o transmitida por un sistema de recuperación de información, en ninguna forma ni por ningún medio, sea mecánico, fotoquímico, electrónico, magnético, electroóptico, por fotocopia o cualquier otro, sin el permiso previo, por escrito, de la editorial.

El contenido de este libro es responsabilidad del autor y no refleja necesariamente la opinión de los editores.

Esta obra se publicó por primera vez en versión impresa, en julio de 2011. 
Dame una mano Ilustración digital

La Universidad Peruana de Ciencias Aplicadas (UPC) agradece a Germán Ruiz Ch. la sesión de su ilustración reproducida en la cubierta. 

A Alida Cáceres, por su amor. 


\section{Contenido}

Agradecimientos $\quad 15$

Prólogo 17

Introducción 21

PRIMERA PARTE 25

A puertas del voto electrónico: un estudio preliminar 25

El pasado siempre fue... 31

Sufragio en el siglo XIX: ¿el quién sin el cómo? 35

Miraflores: el doble estatuto 44

Debate parlamentario: la democracia extendida a las cosas 50

La presentación cotidiana del VE $\quad 60$

$\begin{array}{ll}\text { SEGUNDA PARTE } & 67\end{array}$

Capítulo 1. Una alianza extravagante 67

Capítulo 2. El mapa y el diagnóstico técnico 71

Capítulo 3. De las palabras a las cosas 81

Operaciones de «interesamiento»: la zona excluida 81

Como una pizza delivery: la comodidad 86

La resistencia del papel: el reconocimiento no es asunto público 94

La dificultad de las palabras: lógica versus ingenio 99

Lo electrónico como régimen de gobierno 103 
Capítulo 4. Rematerialización del corazón 109

De centros de información (sinergias)... 109

Al sistema (circulatorio) integrado 112

Capítulo 5. Fabricar estilos de comunicación: eficiencia-bienestar $\quad 115$

Versión 1: transparencia

Versión 2: transacciones 123

Versión 3: democracia electrónica 129

Desalejamiento 134

Capítulo 6. La fragilidad de las «cosas públicas»: civilidad y tutela 139

Ciudad digital inalámbrica 139

Los filtros de lista negra 142

Capítulo 7. De los mapas al modelo: relocalizaciones 149

Epílogo. El caso Pacarán: anacronismo y pensamiento trapero 159

Bibliografía 165 


\section{ÍNDICE DE IMÁGENES}

Imagen 1: Intermediarios centrífugos 32

Imagen 2: Conexiones generales del proyecto 72

Imagen 3: Conexiones más detalladas del proyecto 72

Imagen 4: Atmósfera del A/S 400 en radiografía 75

Imagen 5: Circulación por marcos de referencia en la fabricación de aliados 124

Imagen 6: Fabricación de las cosas públicas 135

Imagen 7: Simetría entre fabricación y desalejamiento 137

Imagen 8: Cobertura de la ciudad inalámbrica 140

\section{ÍNDICE DE TABLAS}

Tabla 1: Trabajo de fabricación de la cuasi solución: seguridad y transparencia

Tabla 2: Prioridades vecinales sobre servicios públicos

Tabla 3: Proyectos tecnológicos inscritos en el presupuesto de apertura (dólares americanos)

Tabla 4: Proceso de una alianza 84

Tabla 5: Asociaciones y antiprogramas 89

Tabla 6: Tecnología como hechos o como fabricaciones 94

Tabla 7: Papel versus correo electrónico $\quad 97$

Tabla 8: Historicidad del portal municipal 118

Tabla 9: Homogeneidad y heterogeneidad de medios y auditorios $\quad 123$

Tabla 10: El menor tutelado $\quad 146$ 
«Sí, mi casa es biológica. En el aire hay un latido suave, un pulso que con los años se ha concertado con el mío». (José Watanabe, Mi casa, 2008)

«El medio es el masaje. Ninguna comprensión de un cambio social y cultural es posible cuando no se conoce la manera como los medios funcionan como ambientes». (Marshall McLuhan, 1967) 


\section{Agradecimientos}

Deseo agradecer por su infinita paciencia a todas las personas que han ofrecido su soporte emocional durante los años que duró la investigación que origina este libro; así como a quienes han participado opinando y revisando, en la medida de sus posibilidades, los borradores finales.

Mis profesores de la especialidad de Sociología de la Facultad de Ciencias Sociales de la Universidad de San Marcos han sido mi primer estímulo académico; en el camino de sus cátedras pude madurar mis ideas. Estoy muy agradecido por los conocimientos legados a Manuel Dammert Ego Aguirre, Tirso Molinari, Jaime Ríos Burga, Sinesio López, César Germaná, Agustín Haya de la Torre, Carolina Ortiz, Zenón de Paz, Dunia Samamé y Zenón Vargas.

Nota aparte merece la amistad cultivada con Guillermo Nugent. Estaré siempre agradecido con él por haber estimulado el hábito de la escritura en mí, y por los buenos consejos.

Recuerdo con gratitud el respaldo de Enrique Saldívar Bocángel, exjefe de la Oficina Nacional de Gobierno Electrónico e Informática (Ongei), de la Presidencia del Consejo de Ministros (PCM). Allí pude realizar durante 2007 unas prácticas profesionales dedicadas exclusivamente a llevar a cabo la investigación de mi tesis de licenciatura.

Agradezco enormemente al Instituto Francés de Estudios Andinos (IFEA), a la Embajada de Francia y a la Asamblea Nacional de Rectores por haber otorgado a la tesis el segundo puesto del Premio Franco-Peruano François Bourricaud para la investigación peruana en ciencias humanas y sociales (2010).

En la Universidad Peruana de Ciencias Aplicadas (UPC) he encontrado un perfecto espacio para expresar y desarrollar libremente mis ideas. Me alegra muchísimo que el Fondo Editorial de la UPC, dirigido por Úrsula Freundt-Thurne, mostrara interés por publicar este libro inicial. 
Esta investigación no habría madurado sin las conversaciones con mis compañeros y buenos amigos de la Facultad de Ciencias Sociales en San Marcos. A todos ellos les debo la confianza depositada en mí, así como haber compartido el valor de comprometerse con la vida académica.

Asimismo, quiero expresar gratitud a mis alumnos en las universidades donde enseño, ya que las críticas más agudas a mis ideas provinieron de sus intervenciones en clase.

Finalmente, no hay manera de compensar a mi familia el apoyo incondicional. El ingenio de mi madre, el esfuerzo de mi padre y la alegría de mi hermana están y estarán siempre presentes. 


\section{Prólogo}

El trabajo de Joaquín Yrivarren es tan innovador como bien escrito. Como ya lo anuncian los epígrafes, hay un deliberado esfuerzo por reunir poesía y tecnología.

Se trata de un esfuerzo distinto del modernismo vanguardista de comienzos del siglo XX, cuyos escritores con frecuencia se limitaban a una celebración estridente de los artefactos novedosos de la época: automóviles, aviones, generadores de electricidad y demás. Un elogio de las máquinas en su carácter restringidamente instrumental. La expresión antaño tan usual, como inocente, «máquina de escribir», da cuenta de la inconmovible alteridad que rodeaba la tecnología. Por un lado, el sujeto y, por el otro, el instrumento. Celebración, sí, pero inseparable de la sospecha de su carácter fantasmagórico, amenazante: ¿y si un día las máquinas se independizan de nosotros y piensan por su cuenta? Nótese que las respuestas a esta incertidumbre jamás consideran la posibilidad de una congregación amigable de máquinas que decidirían hacer un mundo por su cuenta sin inmiscuirse en los asuntos «humanos». Tampoco es considerada la posibilidad de máquinas estudiosas que invitarían a los humanos para un seminario de «antropología», el equivalente simétrico de «tecnología», sobre las costumbres humanas. No, siempre se trata de una autonomía hostil, algo así como el temor de una rebelión de los esclavos contra los amos.

La persistencia de estas fantasías se apoya en una drástica separación de los actores humanos, pertenecientes, por una parte, al mundo de la historia, de la sociedad, y, por otra, al mundo no humano, que abarca otras especies vivientes y seres inanimados. Bruno Latour considera que esta separación tajante es el hecho distintivo de la modernidad ${ }^{1}$. El razonamiento moderno, en consecuencia, consistió en un esfuerzo permanente por separar,

\footnotetext{
1 Bruno Latour. Nunca fuimos modernos. Ensayo de antropología simétrica. Buenos Aires: Editorial Siglo XXI, 2008.
} 
purificar, esas dos instancias, lo humano y lo no humano. De ahí la presencia necesariamente amenazante de máquinas «con vida propia». No obstante, señala el autor mencionado, el resultado fue el inverso: una proliferación de híbridos donde los artefactos técnicos adquirieron una importancia central, en una magnitud sin precedentes en otras civilizaciones.

¿Qué sucedió con las sociedades no modernas? Esta separación no fue, ni es, tan tajante. De hecho, la tendencia es la opuesta, una necesidad de poner nombres o dichos a los más distintos artefactos: camiones, ómnibus, colocar adornos en los aparatos, todo ello para subrayar lo opuesto del ideal moderno: la continuidad entre los actores humanos y los no humanos.

Esta perspectiva relacional es utilizada en el presente estudio de Yrivarren para abordar la cuestión del gobierno electrónico. Estamos acostumbrados a conversar y pensar sobre gobiernos democráticos, tiránicos, oligárquicos, pero no asociamos la función de gobernar con un adjetivo instrumental, tecnológico. La investigación de este libro muestra, sin embargo, que estos actores no humanos estuvieron presentes desde los tiempos en que la elección se hacía con votos que eran un papel blanco doblado en cuatro, hasta la actual manera de estar conectados a internet.

El estudio muestra los escenarios que genera la presencia de una nueva tecnología, las ilusiones y las desconfianzas que suscita, los despliegues de las controversias entre todos los involucrados en el proceso, tanto humanos como no humanos. Históricamente el papel, por ejemplo, fue un intermediario que no logró un ensamblaje estable con los ciudadanos, más todavía cuando el no tener la habilidad de leer y escribir era una descalificación para el ejercicio de la ciudadanía en la elección de autoridades.

Rastrear las trayectorias de los actores que se encuentran en un determinado ensamblaje requiere un agudo trabajo descriptivo, pero, sobre todo, una atención a lo que Yrivarren llama prácticas menudas. En mi opinión aquí está uno de los principales logros del trabajo: hacer notar que una cultura democrática no se forma a partir de los grandes discursos, de esos que hablan a la «Historia» con mayúsculas. Está hecha, por el contrario, de esas prácticas que en el momento no le llaman la atención a nadie: un desacuerdo en una asamblea, dudas sobre cuál será la mejor manera de instalar un sistema de alarmas en el distrito, qué se tiene que mostrar en un desfile... 
De manera sutil el texto nos muestra en sus páginas que la democracia no es una especie de virtud inalcanzable que solamente unos cuantos puros, «educados» estarían en condiciones de ejercer. El resultado va más allá de una sucesión aburrida de nimiedades, como una sociología abstracta gustaría de adjetivar. Es, más bien, un llamado sobre las acciones que hacemos sin pensar porque parecen demasiada poca cosa para ocupar nuestra reflexión.

Este libro propone que la educación en democracia no es un asunto de una específica elaboración doctrinal en primer lugar, como prestar atención a las acciones de las que efectivamente está hecha nuestro día a día.

¿Por qué cambian las prácticas en los gobiernos locales? Sin duda, puede afirmarse que es la búsqueda del bienestar. Pero el término quizá no llega a conectar del todo con una sensación que es inmediata: la comodidad. Usualmente se piensa en la comodidad para describir prácticas ajenas a la institucionalidad. Por ejemplo, la informalidad. Es una concepción en lo fundamental negativa. Aquí nos encontramos, sin embargo, con una manera muy distinta de entender la comodidad. Se trata de una atmósfera donde se conectan la simplicidad de las acciones, el buen trato del cuerpo, la confianza entre los miembros del equipo y el uso de un vocabulario amigable.

Por último, y de no menor importancia que todo lo anterior, el estudio hecho sobre el gobierno electrónico es un caso ejemplar donde la elaboración conceptual y el análisis de la gestión confluyen de una manera armoniosa, sin violentar a los acontecimientos, las personas y el entorno no humano.

Cabe esperar, con fundamento y entusiasmo, más contribuciones innovadoras de Joaquín Yrivarren en la literatura sociológica.

Guillermo Nugent

Sociólogo y psicoterapeuta 


\section{Introducción}

«Es preciso ir un poco más lejos y dotar las cosas de agitación, de incertidumbre y de pasión, es decir, de historicidad».

Bruno Latour

Debo confesar que el sentimiento de malestar que me produce hacer algún trámite o solicitar algún servicio al Estado me ha llevado a escribir este libro. Pienso que podremos estar de acuerdo, estimado lector, con que las colas interminables bajo el sol, los papeleos, los cobros excesivos, los rostros adustos, las coimas, las dilaciones intencionales, las argollas obligatorias, los locales atestados de gente y el angustioso «peloteo» pueden generar en uno un sentimiento de inmensa incomodidad que no se puede reducir a un «capricho personal», sino más bien debe ser atribuido a un vínculo social mal ensamblado. Una persona usualmente se siente cómoda cuando entra en contacto con otra persona que la trata bien, cuando sus deseos y creencias individuales son reconocidos.

Se dice que los servicios públicos son la cara del Estado frente al ciudadano. Si tomamos en cuenta lo anterior, se podría decir que a esa «cara» le resulta totalmente indiferente que haya otra «cara» que solicita un servicio. Convengamos que la palabra «cara» es un referente de quien «yo soy» y un canal esencial para aprender cómo se siente una persona; no resulta por eso descabellado defender que el reconocimiento debería ser un elemento esencial en una democracia. Sin embargo, por lo general experimentar el espacio y los servicios públicos nos deja en claro que la individualidad es algo absolutamente prescindible.

Lógicamente me llamó la atención que algo denominado gobierno electrónico tuviera la ambición de hacerle las cosas públicas más cómodas a la gente, a través de la implementación de ciertas tecnologías de información y comunicación. El conjunto de esta obra está dedicado a examinar cómo se 
incorporan tales tecnologías cargadas de una sensibilidad pública, cuya meta es el reconocimiento de expectativas y deseos individuales. Me interesa que este sea un estudio que favorezca la discusión, por lo que he optado por aterrizarloen la experiencia concreta de la Municipalidad de Miraflores durante el periodo 2003-2006. Esta experiencia ha sido considerada exitosa y pionera en el Perú en cuanto al desarrollo de un gobierno electrónico local. Es pertinente decir que esto cobra especial relevancia en la actualidad, ya que, después de cuatro años de silencio e inactividad, la municipalidad ha vuelto a declarar de interés prioritario el tema. En enero de 2011 aprobó con carácter vinculante la «Carta de gobierno electrónico», documento que pone de manifiesto sus principios y encarga a una comisión especial su dirección. Además, se ha hecho explícito el respaldo de la Oficina Nacional de Gobierno Electrónico.

Quiero facilitar al lector algún entendimiento acerca de lo que conlleva hablar de gobierno electrónico. Principalmente, estos proyectos de innovación tecnológica traducen una agenda de cambios sobre lo que conocemos como burocracia, agenda que data de muchos años atrás². Su pretensión es adaptar las organizaciones públicas al nuevo estilo de sociabilidad emparentado a la proliferación de medios de comunicación audiovisuales e internet. De ahí que en la literatura especializada sea común leer afirmaciones como:

«El nuevo modelo de sociedad requiere organizaciones más abiertas, flexibles, interconectadas, orientadas al funcionamiento en red y con capacidad de reacción inmediata, así como estructuras más planas con menor número de niveles jerárquicos y un mayor grado de comunicación informal». (Martínez y otros 2006: 2)

Para cualquier gestión pública, un tema especialmente importante es la generación, el manejo y la comunicación de información y conocimiento. Tradicionalmente, el papel ha sido el soporte material de la información y aún constituye la materia prima de las oficinas públicas. Sin exagerar, podemos decir que el papel es toda una autoridad en lo que a asuntos públicos se refiere; todavía para muchos funcionarios públicos «papelito manda». De la misma manera, para muchos peruanos «estar empapelado» significa estar sometido a un poder injusto ${ }^{3}$. Son escasas las referencias en que el papel

\footnotetext{
2 Cfr. Criado y Ramilo 2002.

3 Lamentablemente, a uno lo empapelan en una comisaría o en el Poder Judicial, escenarios donde precisamente la justicia debería impartirse con imparcialidad.
} 
es el soporte de la expresión individual o del respeto a las instituciones. En nuestra cultura pública la reunión de un conjunto de papeles suele ser asociada a un expediente judicial antes que a una biblioteca pública ${ }^{4}$. Entre los objetivos del gobierno electrónico está cambiar las relaciones materiales de autoridad; y es en este sentido que se habla de paperless. Curiosamente, en la literatura especializada este tema del paperless es asumido como uno menor, que atañe simplemente a la materialidad de la gestión. Sin embargo, en este estudio será pieza clave.

Entonces, al intentar transformar la burocracia, el gobierno electrónico busca sintonizar con las expectativas de reconocimiento de la gente, así como con sus estilos de comunicación. Sin embargo —en mi opinión-, no hay una definición que haga mérito a su espíritu ${ }^{5}$. La mayoría de la definiciones son esencialmente guías para la planificación; como esta:

«Se define como la utilización de las tecnologías de las TIC por parte de los gobiernos locales a través de la mejora de su gestión interna, de la oferta de servicios e información y de los sistemas de intercambio e interacción con los ciudadanos y con las organizaciones públicas y privadas. Acciones a las que cabe añadir las orientadas a sentar las bases para desarrollar la sociedad de la información, tanto en lo que se refiere a la promoción de infraestructuras como a la promoción de una cultura de la información». (Salvador 2002: 1)

Por otra parte, si se repara en el lema del gobierno electrónico en el Perú, «el Estado al servicio del ciudadano», resulta difícil hacerse alguna idea de la naturaleza de los cambios que propone. Vale decir, la carencia de algún adverbio que sugiera cómo se brindarán los servicios o el modo como se experimentarán los asuntos públicos, pone en evidencia que aún resulta espinoso conjugar en un mismo vocabulario las palabras «tecnología», «comodidad» $\mathrm{y}$ «democracia». Por ejemplo, para un grupo de planificadores del gobierno electrónico podría sonar disparatado que lo electrónico permite experimentar los asuntos públicos cómodamente.

\footnotetext{
4 Cfr. Nugent 1996.

5 Una definición clásica es la del Gartner Group: «The continuous optimisation of Government service delivery, citizen participation and governance by transforming internal and external relationships through technology, the Internet and new media» (Criado y Ramilo 2002: 3, Criado y otros 2002: 5).
} 
Ahora bien, debo añadir que el lector no hallará aquí un estudio del gobierno electrónico cuyo respaldo provenga de la teoría de la administración, del derecho o de las ciencias políticas. Antes bien, el interés académico estriba en la intersección de la sociología de la tecnología y la teoría de la comunicación. Ubicados en esta intersección es posible apreciar, como elementos de un mismo proceso, el trabajo de implementación tecnológica (interconexión de bases de datos, funcionamiento de plataformas web, diseños de portales) y el desarrollo de estilos de comunicación e interacción con los vecinos. Como el epígrafe de esta introducción insinúa, la clave de este estudio está en dotar de historicidad a los medios técnicos de interconexión. El argumento principal de este estudio es que el gobierno electrónico constituye un proceso constante de fabricación (tecnocomunicativa) de las cosas públicas, en que está en juego el desarrollo de una sensibilidad que sintonice con la comodidad de los ciudadanos. Y como queda dicho, la comodidad no es un «capricho personal» sino una forma de hablar de democracia, o sea, del reconocimiento de la individualidad en el gobierno de los asuntos públicos.

Este libro está organizado en dos partes. En primer lugar, el lector encontrará un estudio preliminar acerca del voto electrónico, escrito con ocasión de la decisión del Congreso de la República de dar facultades a la Oficina Nacional de Procesos Electorales (ONPE) para implementar de manera gradual esta nueva técnica de sufragio. El estudio se presenta como un ensayo que explora una interpretación alternativa de nuestra democracia representativa a partir de su materialidad. Después, el libro se compone de siete capítulos, donde se desarrolla la indagación sobre la experiencia de la Municipalidad de Miraflores. El estudio preliminar se puede leer independientemente del resto de capítulos; incluso, en él se compila la mayoría de ideas que se desenvuelven después. Y si el lector estuviera en un apuro, recomiendo leer el capítulo 3 de la segunda parte («De las palabras a las cosas») y el capítulo 5 («Fabricar estilos de comunicación: eficiencia-bienestar»), ya que en ambos se desarrolla mi argumento principal. 


\section{Primera parte}

\section{A PUERTAS DEL VOTO ELECTRÓNICO: UN ESTUDIO PRELIMINAR}

«El recuento de votos, una parte muy apreciada del proceso de fragmentación del siglo XVIII, en un ambiente de velocidades eléctricas instantáneas se ha convertido rápidamente en una forma engorrosa e ineficaz de evaluación social».

Marshall McLuhan

Este estudio preliminar es una deuda pendiente a nivel personal y académico. Su contenido está destinado a mostrar que las tecnologías de sufragio, en especial, el polémico voto electrónico (desde ahora VE), son una ocasión que no deberíamos perder de vista para evaluar la construcción de nuestra democracia representativa. La tecnología electoral no ha sido objeto de interés académico (historiográfico), y solo en la actualidad es motivo de interés público a puertas de la implementación del VE. En rigor, existe un déficit profundo en el conocimiento de las técnicas de representación en nuestro país, conocimiento que es vital para sentir la intensidad del cambio originado por el VE. Y este asunto no es nimio, toda vez que se suma (aunque tangencialmente) a temas cruciales y harto debatidos, como la crisis del sistema de partidos, la legitimidad de las instituciones, la inmunidad parlamentaria, la renovación del Congreso, las reformas constitucionales, etcétera. En fin, una serie de temas que tienen que ver con la evaluación del sistema representativo, cerca ya del bicentenario de la República.

El VE, a diferencia de aquellos debates, nos obliga a pensar sobre la representatividad desde otro enfoque: el de su materialidad y, más aún, la fabricación de esa materialidad. Por su definición,

«El voto electrónico es un sistema de sufragio que utiliza una combinación de procedimientos, con componentes de hardware, software y red de comunicaciones que permiten automatizar los procesos de identificación 
del elector, de emisión del voto, de escrutinio, de conteo de votos, de emisión de reportes y de presentación de resultados, de un proceso electoral, referendo y otras consultas populares». (Yrivarren Lazo 2008: 53)

En este sentido, el VE es algo así como el producto del trabajo arquitectónico, que da forma a una nueva materialidad que servirá de soporte para el ejercicio del sufragio. Por eso, quiero sugerir que lo primero que el VE induce a pensar es que el proceso de representación más elemental de cualquier República democrática está mediado por una materialidad que es susceptible de cambios a través del tiempo. En la Oficina Nacional de Procesos Electorales (ONPE) desde 1996 se inicia el desarrollo del software relacionado con esta nueva arquitectura material. Desde esa época se ha ido automatizando partes de la jornada electoral, siguiendo una tendencia (internacional) hacia la automatización de todo el proceso, desde la identificación hasta la transmisión.

Los ingenieros especializados en VE insisten en decir que este sistema no solo se compone de las máquinas de votación, de la interfaz hombremáquina, ni se limita a la jornada electoral, sino que se trata esencialmente de «optimizar» la etapa previa a la jornada electoral, la jornada misma y su etapa posterior, en virtud de la incorporación de conocimiento, tecnología y experiencia en todo el proceso electoral ${ }^{6}$. Es más, en la actualidad solo falta terminar de automatizar la jornada electoral ${ }^{7}$. Y curiosamente, justo en esta etapa, de mayor notoriedad pública, han empezado a emerger las opiniones a favor del proyecto y en contra de él.

Desde 2002 el proyecto cuenta con una planificación seria y además ese año se inició el diseño de una solución tecnológica, la cual no conllevó la adquisición de una «receta genuina» ni una «fórmula mágica» precisamente, sino más bien el despliegue de un trabajo de innovación en que se identificaron problemas y se buscaron soluciones. Así, tal «solución», que a la sazón era mejor llamarla «cuasi solución», ha estado sometida a una serie de pruebas en diferentes eventos y demostraciones. Por ejemplo, las elecciones internas de partidos políticos, la elección de delegados en municipalidades

\footnotetext{
6 Cfr. Yrivarren Lazo 2004.

7 Según Luis Panizo: «Algo importante que hay que cambiar es la gestión de los procesos electorales electrónicos, porque en este punto es muy importante preguntarse: ¿si vamos a implementar el voto electrónico, tenemos que reemplazar toda nuestra logística? Evidentemente, uno de los mayores errores es no hacerlo. Hay que variar el rol de las personas» (Panizo 2008: 21).
} 
y de representantes en instituciones públicas. Una experiencia emblemática a nivel nacional fue la elección de delegados vecinales en la Municipalidad de Miraflores en 2004 (de la cual hablaremos más adelante). Sin embargo, 2005 marcó un hito en el proyecto dirigido desde la ONPE, pues la Ley 28581 autorizó a esta institución implementar el VE de manera gradual y progresiva. Naturalmente, así como a nadie le gustaría despertarse un día y hallar que su casa fue demolida y que a un grupo de arquitectos se le ocurrió construirle otra, tampoco nadie se sentiría a gusto con un sistema electoral cuya materialidad cambie abruptamente. Ese mismo año, el VE fue incluido como una acción estratégica del Plan de Desarrollo Nacional de la Sociedad de la Información en el Perú (Agenda Digital). Y un año después sería incorporado en la Estrategia Nacional de Gobierno Electrónico e Informática. De suerte que el VE es un elemento fundamental si se quiere pensar la sociedad de la información en el Perú.

Para la ONPE, la fabricación de esta técnica de representación está guiada por cuatro pilares:

1. La seguridad, que implica crear garantías técnicas de funcionamiento.

2. La transparencia, que se basa en el «enrolamiento» de todos los actores involucrados en la generación de confianza colectiva.

3. La usabilidad, que se enfoca en la facilidad y comodidad de uso de los dispositivos y las interfaces hombre-máquina.

4. La accesibilidad, lo que implica una tecnología no excluyente frente a la diversidad geográfica, demográfica y social del país.

En cuanto a la usabilidad y la accesibilidad, se ha previsto que el prototipo de la máquina de votación (y de sus dispositivos accesorios) tenga autonomía energética y que su diseño permita su fácil traslado y conservación. Asimismo, por medio de diferentes pruebas, se han buscado alternativas para salvar la dificultad que impone diseñar una interfaz material y gráfica «amigable». Sin duda, los problemas de extensión y distribución (acceso), así como la creación de usuarios competentes, son puntos axiales desde donde evaluar la brecha digital en el país. El VE se incorpora como un proceso de aprendizaje necesario respecto a cómo ejercer la ciudadanía en un nuevo ambiente material, en este caso electrónico y virtual. Más específicamente, crearía un «hábitat» digital conforme a un cambio generacional que diferencia cada vez más entre nativos e inmigrantes digitales. 
TABLA 1: TRABAJO DE FABRICACIÓN DE LA CUASI SOLUCIÓN: SEGURIDAD Y TRANSPARENCIA

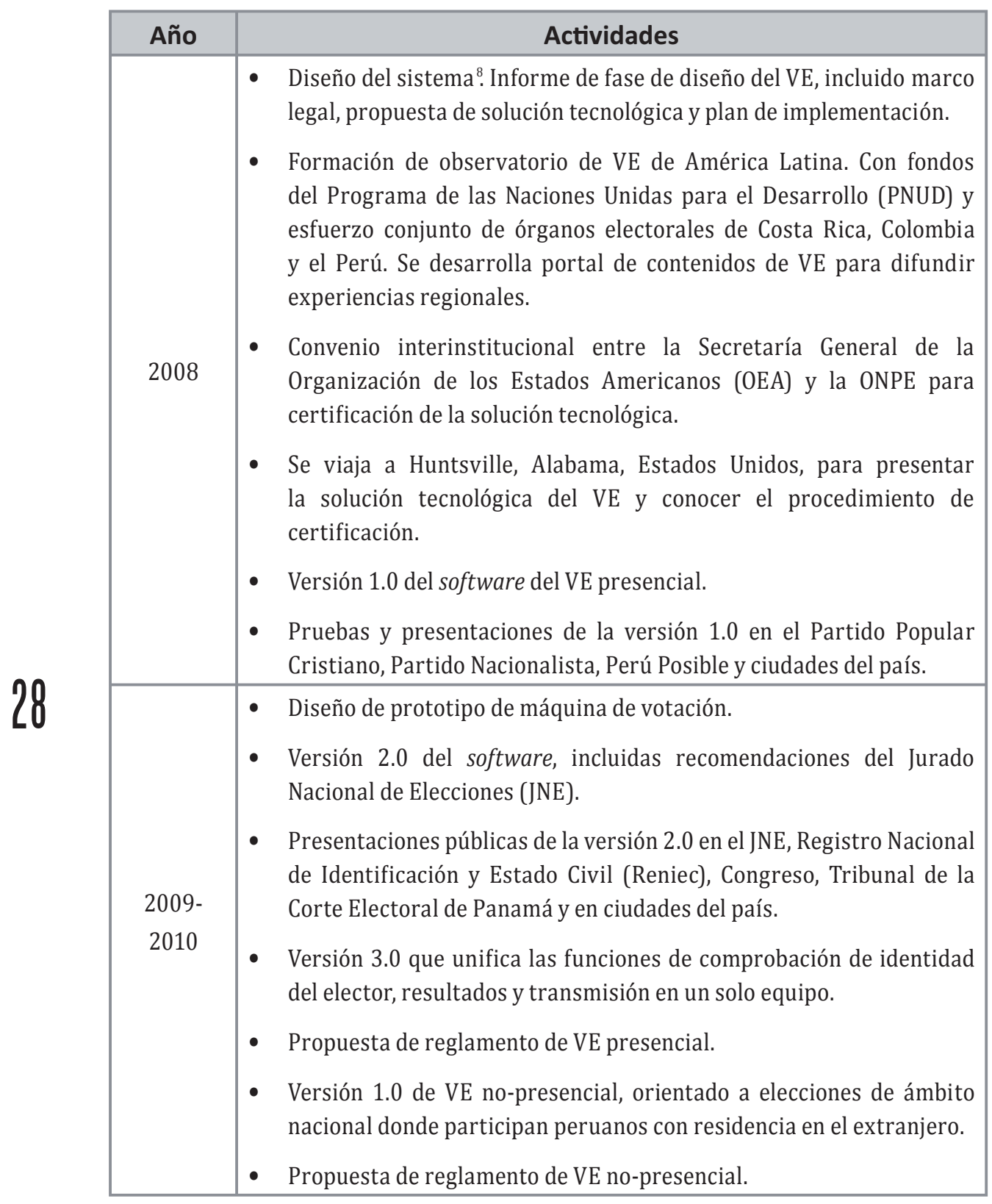

8 El diseño del sistema de votación presencial implicó tomar una serie de decisiones acerca del sistema operativo, lenguaje de programación, archivo de texto para el almacenamiento de votos y votantes, arquitectura de operación, almacenamiento USB y smart card, medios de transmisión de resultados. 


\begin{tabular}{|c|l|}
\hline Año & \multicolumn{1}{c|}{ Actividades } \\
\hline & • $\begin{array}{l}\text { Facultad de Ingeniería Industrial de la Universidad Nacional Mayor } \\
\text { de San Marcos (UNMSM) fabrica máquina de votación. Problemas } \\
\text { técnicos relativos con el tiempo de entrega. }\end{array}$ \\
$2009-$ & $\begin{array}{l}\text { Adecuación de la versión } 3.0 \text { del software con la versión } 1.0 \text { del } \\
\text { prototipo de la máquina de votación. }\end{array}$ \\
& $\begin{array}{l}\text { ONPE recibe certificación de proyectos de VE presencial y no- } \\
\text { presencial. La certificación da fe de que el proceso está según lo } \\
\text { establecido en la NTP ISO/IEC 12207. } \\
\text { Ceremonia pública en el Instituto para la Calidad de la PUCP. }\end{array}$ \\
\hline
\end{tabular}

Elaboración propia.

Si bien el VE está incluido en los planes y estrategias de ciertas instituciones cuya labor es sentar las bases de la sociedad de la información, ello no garantiza que el VE sea la «solución tecnológica» deseada. En general, en el Perú los marcos institucionales no son la base de la innovación y del ingenio; más bien, la creatividad es el mecanismo cotidiano para escapar de los marcos que nos constriñen y nos limitan ${ }^{9}$. De ahí que una solución tecnológica sea el producto de un proceso de fabricación como el que es descrito esquemáticamente en el cuadro de arriba (para un periodo reducido de tres años). Se puede observar que el proceso de fabricación de la solución tecnológica VE opera un tránsito desde un simple informe de diseño del sistema hasta la versión 3.0 del software ya certificado e integrado con la versión 1.0 del hardware. Pero, en este tránsito, es fundamental identificar la cantidad de asociaciones establecidas entre elementos tan heterogéneos como informes, decisiones, softwares, instituciones nacionales, internacionales, hardwares, plataformas web, certificados, universidades, auditorios, fuentes de financiamiento, reglamentos, y así. El caso es que el proceso de fabricación del VE lleva a describir versiones sucesivas de una «cuasi solución» a partir de sus múltiples y variadas asociaciones. Así, nombrar una solución tecnológica de VE supone llegar a un punto más o menos estable del proceso de fabricación, punto que resume el conglomerado sociotécnico que le da vida.

Mi sensación es que por el modo como se ha conducido el proceso de fabricación de la solución tecnológica del VE, esta no podría ser calificada

$9 \quad$ Cfr. Yrivarren 2011. 
de «caja negra»; como si fuera una tecnología cerrada al escrutinio público, e impuesta por alguna entidad gubernamental. Antes bien, el trabajo sobre el VE ha sido más parecido a la actividad del maestro de obra que hace artesanalmente una casa de uno o más pisos, que al chasquido de un genio que aparece un departamento ya amoblado de la nada. En su labor, el maestro puede encontrarse con errores, así como un ingeniero del VE seguramente tendrá que afrontar muchos; ello no debería generar un sentimiento de sospecha y consternación, sino antes bien una afirmación en la capacidad de ingenio y adaptación que lleva a rediseñar la estructura de la casa sobre la marcha.

Una característica importante del modo como se ha desarrollado el VE en el Perú es haber hecho visible el tejido de sus asociaciones, a las que también podemos denominar alianzas tecnoinstitucionales destinadas a generar un clima de confianza y auditoría constante sobre la tecnología puesta a prueba. Al hilo de esto, la tecnología ha sido desarrollada (software y hardware) enteramente en el Perú (in house), lo cual evita tener que guardar el celo de proveedores en cuanto a la información que habría de lindar con su propiedad intelectual. Según P. Medina:

«Amparados en leyes de propiedad intelectual, así como en el propio celo, los proveedores tratan de brindar la menor cantidad de información incluso al órgano electoral, y menos aún a los partidos políticos y técnicos independientes, con lo cual se genera la sensación de que esto es una caja negra que nadie puede auditar». (2008: 86)

Seguramente alguna vez usted ha escuchado decir la frase «dime con quién andas y te diré quién eres», usada para insinuar que una persona puede ser descrita y juzgada por la calidad de sus amigos o «socios». Mi punto de vista es que esta frase popular puede y debe ser aplicada al VE, siempre que su evaluación u observación asuma la perspectiva de la fabricación sociotécnica de la tecnología. Un punto central para el desenvolvimiento de una observación orientada a la generación de confianza ${ }^{10}$ es la descripción del proceso de fabricación de la tecnología y la consecuente formación de alianzas tecnoinstitucionales. Un antecedente inmediato es la forma de evaluación de las candidatas a la Municipalidad de Lima (2010), donde a partir del conocimiento del cruce de los círculos sociales ${ }^{11}$ se podía juzgar su proximidad, por ejemplo, a redes de narcotráfico o corrupción. Así, la descripción de asociaciones, cruces y alianzas constituye un canal valioso para crear confianza.

10 Cfr. Durand 2008, Medina 2008, y Nunes 2008.

11 Cfr. Simmel 1972b. 
Para poder revisar todo el contenido de esta edición, visite nuestra tienda virtual.

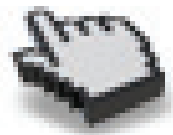

Gobierno electrónico

Análisis de los conopptos de tenologia, comodidad y democracia

Joaquin Yrivarren Espinoza

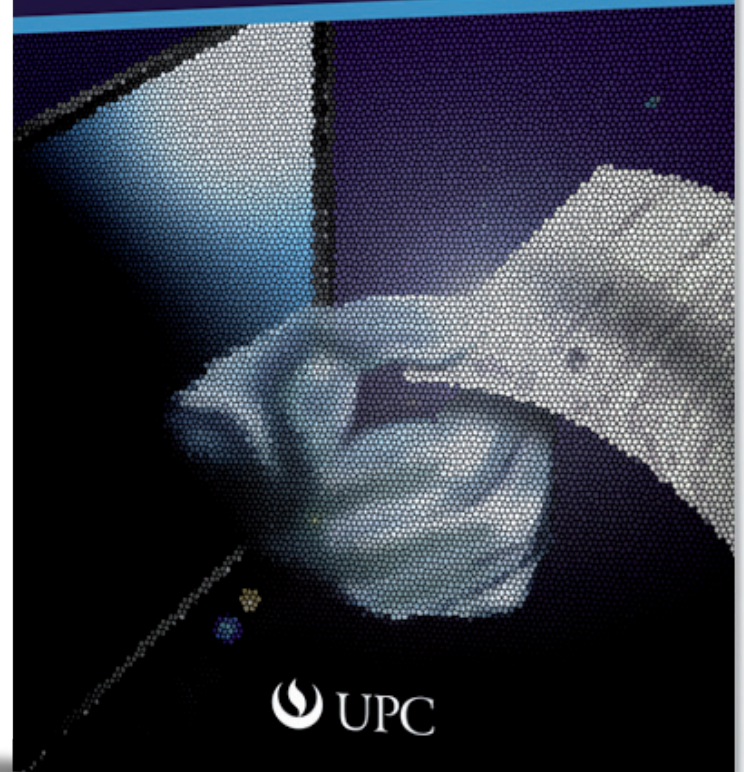

